EPJ Web of Conferences 112,02001 (2016)

DOI: $10.1051 /$ epjconf/20161120200 1

(C) Owned by the authors, published by EDP Sciences, 2016

\title{
CGC beyond eikonal accuracy: finite width target effects
}

\author{
Tolga Altinoluk ${ }^{1,2, a}$, Nestor Armesto ${ }^{1}$, Guillaume Beuf ${ }^{3}$, and Alexis Moscoso ${ }^{1}$ \\ ${ }^{1}$ Departamento de Física de Partículas and IGFAE, Universidade de Santiago de Compostela, E-15706 \\ Santiago de Compostela, Galicia-Spain \\ ${ }^{2}$ CENTRA, Instituto Superior Técnico, Universidade de Lisboa, Av. Rovisco Pais, P-1049-001 Lisboa, Por- \\ tugal \\ ${ }^{3}$ European Centre for Theoretical Studies in Nuclear Physics and Related Areas (ECT*) and Fondazione \\ Bruno Kessler, Strada delle Tabarelle 286, I-38123 Villazzano (TN), Italy
}

\begin{abstract}
We present a method to systematically include the corrections to the eikonal approximation that are associated with the finite width of the target. The retarded gluon propagator in background field is calculated at next-to-next-to-eikonal (NNE) accuracy by using this method. The corrections to the strict eikonal limit of the gluon propagator are found to be Wilson lines decorated by gradients of the background field of the target. The result is then applied to single inclusive gluon production and to single transverse spin asymmetry for a polarized target in pA collisions.
\end{abstract}

\section{Introduction}

The high energy limit of the dilute-dense scattering processes adopts two key approximations within the Color Glass Condensate (CGC, see [1] and references therein) effective theory. The first approximation is called the semi-classical approximation and it amounts to replacing the target by an intense classical background field. The other one is referred to as eikonal approximation, in which the constituents of the projectile just experience color rotation upon scattering with the target, picking a Wilson line at a given transverse point but integrated along the light-cone direction of propagation of the projectile, for which the target appears as infinitely Lorentz contracted. Corrections suppressed by inverse powers of the energy of the collision are systematically neglected within the eikonal limit. We study such power suppressed corrections to the CGC, namely next-to-eikonal contributions due to finite width of the target $[2,3]$. A similar analysis in the context of soft gluon resummation for hard scattering amplitudes is performed in $[4,5]$.

\section{Eikonal expansion of the retarded gluon propagator in background field}

One of the most important elements to describe the dilute-dense scattering processes at high energy is the retarded gluon propagator in a classical background field. A highly boosted left-moving target can be described by a classical background gluon field $\mathcal{A}_{a}^{-}\left(x^{+}, \mathbf{x}\right)$. Only the (-) component of this field is enhanced by a Lorentz gamma factor, so the other components are negligible. Moreover, one can also

\footnotetext{
ae-mail: tolga.altinoluk@usc.es
} 
neglect the $x^{-}$dependence of this field due to time dilation. In the presence of such a field, it is natural to work in the light-cone gauge $\mathcal{A}_{a}^{+}=0$. Since the background field is independent of $x^{-}$component, it is convenient to introduce the one dimensional Fourier transform of the retarded gluon propagator

$$
G_{R}^{\mu v}(x, y)_{a b}=\int \frac{\mathrm{d} p^{+}}{2 \pi} e^{-i k^{+}\left(x^{-}-y^{-}\right)} \frac{1}{2\left(k^{+}+i \epsilon\right)} \mathcal{G}_{k^{+}}^{\mu v}(\underline{x} ; \underline{y})_{a b} .
$$

For our purposes we only need the $(i-)$ component of the retarded gluon propagator which is written in terms of the background scale propagator as

$$
\mathcal{G}_{k^{+}}^{i-}\left(\underline{x}, \underline{y}^{a b}=\frac{i}{k^{+}+i \epsilon} \partial_{\mathbf{y}^{\mathbf{y}}} \mathcal{G}_{k^{+}}^{a b} \underline{x}, \underline{y}\right)
$$

with the notation $\underline{x} \equiv\left(x^{+}, \mathbf{x}\right), \mathbf{x}$ being the transverse component. The scalar propagator $\mathcal{G}_{k^{+}}^{a b}(\underline{x}, \underline{y})$ is the retarded solution of the Green's equation

$$
\left[\delta^{a b}\left(i \partial_{x^{+}}+\frac{\partial_{\mathbf{x}}^{2}}{2\left(k^{+}+i \epsilon\right)}\right)+g\left(\mathcal{A}^{-}(\underline{x}) \cdot T\right)^{a b}\right] \mathcal{G}_{k^{+}}^{b c}(\underline{x} ; \underline{y})=i \delta^{a c} \delta^{(3)}(\underline{x}-\underline{y}) .
$$

The scalar Green's equation (3) has a form of a Schrodinger equation in $2+1$ dimensions with a matrix potential. Its solution can thus be written as a path integral [6-8]. For our purposes, we need the discretized form of the path integral, which reads

$$
\mathcal{G}_{k^{+}}^{a b}(\underline{x}, \underline{y})=\lim _{N \rightarrow \infty} \int\left(\prod_{n=1}^{N-1} d^{2} \mathbf{z}_{n}\right)\left[\prod_{n=0}^{N-1} \mathcal{G}_{0, k^{+}}\left(z_{n+1}^{+}, \mathbf{z}_{n+1} ; z_{n}^{+}, \mathbf{z}_{n}\right)\right] \mathcal{U}^{a b}\left(x^{+}, y^{+} ;\left\{\mathbf{z}_{n}\right\}\right)
$$

with the boundary conditions $\mathbf{z}_{0} \equiv \mathbf{y}$ and $\mathbf{z}_{N} \equiv \mathbf{x}$, and

$$
z_{n}^{+}=y^{+}+\frac{n}{N}\left(x^{+}-y^{+}\right) .
$$

The free scalar propagator appearing in the path integral expression, given in equation (4) reads

$$
\mathcal{G}_{0, k^{+}}(\underline{x}, \underline{y})=\theta\left(x^{+}-y^{+}\right)\left(\frac{-i k^{+}}{2 \pi\left(x^{+}-y^{+}\right)}\right) \exp \left(\frac{i k^{+}}{2\left(x^{+}-y^{+}\right)}(\mathbf{x}-\mathbf{y})^{2}\right) .
$$

The discretized Wilson line is defined as

$$
\mathcal{U}^{a b}\left(x^{+}, y^{+},\left\{\mathbf{z}_{n}\right\}\right)=\mathcal{P}_{+}\left\{\prod_{n=0}^{N-1} \exp \left[i g \frac{\left(x^{+}-y^{+}\right)}{N}\left(\mathcal{A}^{-}\left(z_{n}^{+}, \mathbf{z}_{n}\right) \cdot T\right)\right]\right\}^{a b},
$$

with $\mathcal{P}_{+}$denoting path ordering along the $x^{+}$direction.

In the expressions of the observables related to the dilute-dense scattering at high energy, the background propagator appears typically through its Fourier transform $\int d^{2} \mathbf{x} e^{-i \mathbf{k} \cdot \mathbf{x}} \mathcal{G}_{k^{+}}^{a b}(\underline{x}, \underline{y})$. In order to study the corrections to the eikonal limit, we define the medium modification factor $\widetilde{\mathcal{R}}_{\underline{k}}^{a b}\left(x^{+}, y^{+} ; \mathbf{x}\right)$ as

$$
\left.\left.\int d^{2} \mathbf{x} e^{-i \mathbf{k} \cdot \mathbf{x}} \mathcal{G}_{k^{+}}^{a b} \underline{x}, \underline{y}\right)=\widetilde{\mathcal{R}}_{\underline{k}}^{a b}\left(x^{+}, y^{+} ; \mathbf{y}\right) \int d^{2} \mathbf{x} e^{-i \mathbf{k} \cdot \mathbf{x}} \mathcal{G}_{0, k^{+}} \underline{x}, \underline{y}\right) .
$$

In order to calculate the expansion of $\widetilde{\mathcal{R}}_{\underline{k}}^{a b}\left(x^{+}, y^{+} ; \mathbf{x}\right)$ beyond the eikonal approximation, it is more convenient to consider the large $k^{+}$limit than the limit of the large boost of the target. The two limits of course equivalent due to the invariance of the whole scattering process under longitudinal boosts. 
As it is discussed in details in [2,3], the large $k^{+}$expansion of the medium modification factor is performed in two steps. First, the $k^{+}$expansion is calculated for fixed $\mathbf{k} / k^{+}$(semi-classical expansion) and then the result is re-expanded for small $\mathbf{k} / k^{+}$(small angle expansion). At the end, after performing both the semi-classical and the small angle expansion, the medium modification factor $\widetilde{\mathcal{R}}_{\underline{k}}^{a b}\left(x^{+}, y^{+} ; \mathbf{x}\right)$ reads

$$
\begin{aligned}
& \widetilde{\mathcal{R}}_{\underline{k}}\left(x^{+}, y^{+} ; \mathbf{y}\right)=\mathcal{U}\left(x^{+}, y^{+} ; \mathbf{y}\right)+\frac{\left(x^{+}-y^{+}\right) \mathbf{k}^{i}}{k^{+}} \mathcal{U}_{[0,1]}^{i}\left(x^{+}, y^{+} ; \mathbf{y}\right)+i \frac{\left(x^{+}-y^{+}\right)}{2 k^{+}} \mathcal{U}_{[1,0]}\left(x^{+}, y^{+} ; \mathbf{y}\right) \\
& +\frac{\left(x^{+}-y^{+}\right)^{2} \mathbf{k}^{i} \mathbf{k}^{j}}{\left(k^{+}\right)^{2}} \mathcal{U}_{[0,2]}^{i j}\left(x^{+}, y^{+} ; \mathbf{y}\right)+i \frac{\left(x^{+}-y^{+}\right)^{2} \mathbf{k}^{i}}{2\left(k^{+}\right)^{2}} \mathcal{U}_{[1,1]}^{i}\left(x^{+}, y^{+} ; \mathbf{y}\right)-\frac{\left(x^{+}-y^{+}\right)^{2}}{4\left(k^{+}\right)^{2}} \mathcal{U}_{[2,0]}\left(x^{+}, y^{+} ; \mathbf{y}\right) \\
& +O\left(\left(\frac{\left(x^{+}-y^{+}\right)|\mathbf{k}|}{k^{+}} \partial_{\perp}\right)^{3}\right)+O\left(\left(\frac{\left(x^{+}-y^{+}\right)}{k^{+}} \partial_{\perp}^{2}\right)^{3}\right)
\end{aligned}
$$

Here $\mathcal{U}\left(x^{+}, y^{+} ; \mathbf{y}\right)$ is the usual Wilson line operator and $\mathcal{U}_{[i, j]}\left(x^{+}, y^{+} ; \mathbf{y}\right)$ are the decorated Wilson line operators that appear beyond the strict eikonal limit. For the details of the calculation and the explicit expressions of the decorated Wilson lines, we refer to the reader [3].

\section{Single inclusive gluon production in pA collisions}

In the CGC formalism, a highly boosted left-moving nucleus is usually described by a classical gluon shockwave $\mathcal{A}_{a}^{\mu}(x)=\delta^{\mu-} \delta\left(x^{+}\right) \mathcal{A}_{a}^{-}(\mathbf{x})$ in the light-cone gauge $A_{a}^{+}=0$. That field has indeed a vanishing longitudinal width and no $x^{-}$dependence in the limit of infinite boost.

Consider instead a background field

$$
\mathcal{A}_{a}^{\mu}(x)=\delta^{\mu-} \mathcal{A}_{a}^{-}\left(x^{+}, \mathbf{x}\right)
$$

with a finite support along the $x^{+}$direction, from $x^{+}=0$ to $x^{+}=L^{+}$. In the case of a large nucleus, this should be the dominant finite-boost correction with respect to the usual gluon shockwave. On the other hand, a highly boosted right-moving proton, considered as dilute, is described by a classical color current

$$
j_{a}^{\mu}(x)=\delta^{\mu-} j_{a}^{+}(x)
$$

with zero width along $x^{-}: j_{a}^{+}(x) \propto \delta\left(x^{-}\right)$. Let us consider a proton-nucleus collision with a particular impact parameter $\mathbf{B}$ and choose the center of the nucleus as the reference point for the transverse plane, so that a generic point $\mathbf{x}$ in the transverse plane is at a distance $|\mathbf{x}-\mathbf{B}|$ from the center of the proton and at a distance $|\mathbf{x}|$ from the center of the nucleus.Then, the color current $j_{a}^{+}(x)$ can be written as $j_{a}^{+}(x)=\delta\left(x^{-}\right) \mathcal{U}^{a b}\left(x^{+},-\infty, \mathbf{x}\right) \rho^{b}(\mathbf{x}-\mathbf{B})$ where $\rho^{b}$ is the transverse density of color charges inside the proton before it reaches the nucleus, and $\mathcal{U}^{a b}\left(x^{+},-\infty, \mathbf{x}\right)$ is the Wilson line implementing the color precession of these color charges in the background field $\mathcal{A}_{a}^{-}\left(x^{+}, \mathbf{x}\right)$ of the nucleus. One can define the Fourier transform of the color charge density

$$
\rho^{a}(\mathbf{y}-\mathbf{B})=\int \frac{d^{2} \mathbf{q}}{(2 \pi)^{2}} e^{i \mathbf{q} \cdot(\mathbf{y}-\mathbf{B})} \tilde{\rho}(\mathbf{q})
$$

and the gluon-nucleus reduced amplitude, $\overline{\mathcal{M}}_{\lambda}^{a b}(\underline{k}, \mathbf{q})$, as

$$
\mathcal{M}_{\lambda}^{a}(\underline{k}, \mathbf{B})=\int \frac{d^{2} \mathbf{q}}{(2 \pi)^{2}} e^{-i \mathbf{q} \cdot \mathbf{B}} \overline{\mathcal{M}}_{\lambda}^{a b}(\underline{k}, \mathbf{q}) \tilde{\rho}^{b}(\mathbf{q}),
$$


where the gluon production amplitude is $\mathcal{M}_{\lambda}^{a}(\underline{k}, \mathbf{B})$ is given as

$$
\mathcal{M}_{\lambda}^{a}(\underline{k}, \mathbf{B})=\epsilon^{i *}\left(2 k^{+}\right) \lim _{x^{+} \rightarrow \infty} \int d^{2} \mathbf{x} \int d x^{-} e^{i k \cdot x} \int d^{4} y G_{R}^{i-}(x, y)_{a b} j_{b}^{+}(y) .
$$

The cross section for single inclusive gluon production can be written as

$$
k^{+} \frac{d \sigma}{d k^{+} d^{2} \mathbf{k}}=\int \frac{d^{2} \mathbf{q}}{(2 \pi)^{2}} \varphi_{p}(\mathbf{q}) \frac{\mathbf{q}^{2}}{4} \frac{1}{N_{c}^{2}-1} \sum_{\lambda \text { phys. }}\left\langle\overline{\mathcal{M}}_{\lambda}^{a b}(\underline{k}, \mathbf{q})^{\dagger} \overline{\mathcal{M}}_{\lambda}^{a b}(\underline{k}, \mathbf{q})\right\rangle_{A},
$$

where $\varphi_{p}(\mathbf{q})$ is the unintegrated gluon distribution. By using the expanded expression of the medium modification factor one can write the reduced gluon-nucleus amplitude at next-to-next-to-eikonal accuracy as

$$
\begin{aligned}
& \overline{\mathcal{M}}_{\lambda}^{a b}(\underline{k} ; \mathbf{q})=i \varepsilon_{\lambda}^{i *} \int d^{2} \mathbf{y} e^{i \mathbf{y} \cdot(\mathbf{q}-\mathbf{k})}\left\{2\left(\frac{\mathbf{k}^{i}}{\mathbf{k}^{2}}-\frac{\mathbf{q}^{i}}{\mathbf{q}^{2}}\right) \mathcal{U}\left(L^{+}, 0 ; \mathbf{y}\right)\right. \\
& +\left(\frac{L^{+}}{k^{+}}\right)\left[\left(\delta^{i j}-2 \frac{\mathbf{q}^{i} \mathbf{k}^{j}}{\mathbf{q}^{2}}\right) \mathcal{U}_{[0,1]}^{j}\left(L^{+}, 0 ; \mathbf{y}\right)-i \frac{\mathbf{q}^{i}}{\mathbf{q}^{2}} \mathcal{U}_{[1,0]}\left(L^{+}, 0 ; \mathbf{y}\right)\right] \\
& +\left(\frac{L^{+}}{k^{+}}\right)^{2}\left[-2 \frac{\mathbf{q}^{i}}{\mathbf{q}^{2}} \mathbf{k}^{j} \mathbf{k}^{l} \mathcal{U}_{[0,2]}^{j l}\left(L^{+}, 0 ; \mathbf{y}\right)-i \frac{\mathbf{q}^{i} \mathbf{k}^{j}}{\mathbf{q}^{2}} \mathcal{U}_{[1,1]}^{j}\left(L^{+}, 0 ; \mathbf{y}\right)+\frac{1}{2} \frac{\mathbf{q}^{i}}{\mathbf{q}^{2}} \mathcal{U}_{[2,0]}\left(L^{+}, 0 ; \mathbf{y}\right)\right. \\
& \left.\left.+\frac{i}{4}\left(\mathbf{k}^{2} \delta^{i j}-2 \mathbf{k}^{i} \mathbf{k}^{j}\right) \mathcal{U}_{(\mathrm{A})}^{j}\left(L^{+}, 0 ; \mathbf{y}\right)+\frac{\mathbf{k}^{j}}{4} \mathcal{U}_{(\mathrm{B})}^{i j}\left(L^{+}, 0 ; \mathbf{y}\right)+\frac{i}{4} \mathcal{U}_{(\mathrm{C})}^{i}\left(L^{+}, 0 ; \mathbf{y}\right)\right]\right\}^{a b} .
\end{aligned}
$$

After squaring the reduced gluon-nucleus amplitude without summing over the helicity $\lambda$, one can realise that the transverse momentum structure of the strict eikonal and next-to-next-to-eikonal terms are symmetric under the exchange of $i \leftrightarrow j$ where $i$ and $j$ are the indices of the transverse components of the physical polarisation vectors in the amplitude and complex conjugate amplitude. On the other hand, the transverse momentum structure of the next-to-eikonal terms is anti-symmetric under the same exchange.

In the calculation of the single inclusive gluon production cross section one should sum over the gluon polarisations, which leads to

$$
\sum_{\lambda} \epsilon_{\lambda}^{i *} \epsilon_{\lambda}^{j}=\delta^{i j}
$$

Since the transverse momentum structure of the next-to-eikonal terms are anti-symmetric under the exchange of $i \leftrightarrow j$, this contribution to the single inclusive gluon production cross section vanishes. The final expression for the single inclusive gluon cross section reads at next-to-next-to-eikonal accuracy

$$
\begin{aligned}
& k^{+} \frac{d \sigma}{d k^{+} d^{2} \mathbf{k}}=\int \frac{d^{2} \mathbf{q}}{(2 \pi)^{2}} \varphi(\mathbf{q}) \frac{\mathbf{q}^{2}}{4} \int d^{2} \mathbf{r} e^{i \mathbf{r} \cdot(\mathbf{q}-\mathbf{k})}\left\{4 C^{i}(\mathbf{k}, \mathbf{q}) C^{j}(\mathbf{k}, \mathbf{q}) O(\mathbf{r})+\left(\frac{L^{+}}{k^{+}}\right)^{2}\left[2 \frac{\mathbf{q}^{i}}{\mathbf{q}^{2}} C^{i}(\mathbf{k}, \mathbf{q})\right.\right. \\
& \times\left[-2 \mathbf{k}^{l} \mathbf{k}^{m} O_{[0,2]}^{l, m}(\mathbf{r})-2 i \mathbf{k}^{l} O_{[1,1]}^{l}(\mathbf{r})+O_{[2,0]}(\mathbf{r})\right]+C^{i}(\mathbf{k}, \mathbf{q})\left[i\left(\mathbf{k}^{2} \delta^{i l}-2 \mathbf{k}^{i} \mathbf{k}^{l}\right) O_{(\mathrm{A})}^{l}(\mathbf{r})+\mathbf{k}^{m} O_{(\mathrm{B})}^{i m}(\mathbf{r})\right. \\
& \left.\left.+i O_{(\mathrm{C})}^{i}(\mathbf{r})\right]+\tilde{C}^{l i}(\mathbf{k}, \mathbf{q})\left[\tilde{C}^{l i}(\mathbf{k}, \mathbf{q}) O_{[0,1] ;[0,1]}^{l ; m}(\mathbf{r})+2 i \frac{\mathbf{q}^{i}}{\mathbf{q}^{2}} O_{[0,1] ;[1,0]}^{l}(\mathbf{r})\right]+\frac{1}{\mathbf{q}^{2}} O_{[1,0] ;[1,0]}(\mathbf{r})\right\}
\end{aligned}
$$


where $C^{i}(\mathbf{k}, \mathbf{q})=\left(\frac{\mathbf{k}^{i}}{\mathbf{k}^{2}}-\frac{\mathbf{q}^{i}}{\mathbf{q}^{2}}\right), \tilde{C}^{i j}(\mathbf{k}, \mathbf{q})=\left(\delta^{i j}-2 \mathbf{k}^{i} \frac{\mathbf{q}^{j}}{\mathbf{q}^{2}}\right)$ and the dipole operators are defined as

$$
\begin{aligned}
O(\mathbf{r}) & =\int d^{2} \mathbf{b} \frac{1}{N_{c}^{2}-1}\left\langle\operatorname{tr}\left[\mathcal{U}\left(\mathbf{b}+\frac{\mathbf{r}}{2}\right) \mathcal{U}^{\dagger}\left(\mathbf{b}-\frac{\mathbf{r}}{2}\right)\right]\right\rangle_{A}, \\
O_{[\alpha, \beta]}^{i \cdots j}(\mathbf{r}) & =\int d^{2} \mathbf{b} \frac{1}{N_{c}^{2}-1}\left\langle\operatorname{tr}\left[\mathcal{U}_{[\alpha, \beta]}^{i \cdots j}\left(\mathbf{b}+\frac{\mathbf{r}}{2}\right) \mathcal{U}^{\dagger}\left(\mathbf{b}-\frac{\mathbf{r}}{2}\right)\right]\right\rangle_{A}, \\
O_{[\alpha, \beta] ;[\gamma, \delta]}^{i \cdots j ; \cdots m}(\mathbf{r}) & =\int d^{2} \mathbf{b} \frac{1}{N_{c}^{2}-1}\left\langle\operatorname{tr}\left[\mathcal{U}_{[\alpha, \beta]}^{i \cdots j}\left(\mathbf{b}+\frac{\mathbf{r}}{2}\right) \mathcal{U}_{[\gamma, \delta]}^{\cdots \cdots \cdots}\left(\mathbf{b}-\frac{\mathbf{r}}{2}\right)\right]\right\rangle_{A} .
\end{aligned}
$$

Recently, the decorated Wilson lines that appear beyond the eikonal limit have been calculated explicitly to leading order in the field of the target [9]. These corrections, indeed, determine the next-toeikonal and next-to-next-to-eikonal corrections to the Lipatov vertex.

\section{SIngle transverse spin asymmetry: polarized target}

Another interesting observable that one can discuss beyond the eikonal limit is the single transverse spin asymmetry (SSA) for the process $p+A^{\uparrow} \rightarrow g+X$ i.e. with a transversely polarized target. The SSA is defined as

$$
A_{N}=\frac{k^{+} \frac{d \sigma^{\uparrow}}{d k^{+} d^{2} \mathbf{k}}-k^{+} \frac{d \sigma^{\downarrow}}{d k^{+} d^{2} \mathbf{k}}}{k^{+} \frac{d \sigma^{\uparrow}}{d k^{+} d^{2} \mathbf{k}}+k^{+} \frac{d \sigma^{\downarrow}}{d k^{+} d^{2} \mathbf{k}}}=\frac{k^{+} \frac{d \sigma^{\uparrow}}{d k^{+} d^{2} \mathbf{k}}-k^{+} \frac{d \sigma^{\downarrow}}{d k^{+} d^{2} \mathbf{k}}}{2 k^{+} \frac{d \sigma}{d k^{+} d^{2} \mathbf{k}}}
$$

where the denominator corresponds to the unpolarized cross section.

Within the CGC framework, the dependence on the transverse spin vector s should appear through the probability distribution for the background field that leads to s-dependent target averaged quantities, i.e. s-dependent standard and decorated adjoint dipoles. The transverse rotational symmetry around the center of the target still holds for the s-dependent decorated dipoles. Hence, under the transformation $\mathbf{r} \rightarrow-\mathbf{r}$ and $\mathbf{s} \rightarrow-\mathbf{s}$, the decorated dipole operators with even number of transverse indices are invariant and the ones with odd number of indices flip sign.

Due to transverse rotational symmetry around the center of the target, one gets

$$
O(-\mathbf{r},-\mathbf{s})=O(\mathbf{r}, \mathbf{s})
$$

Moreover, the dipole operator satisfies

$$
O(-\mathbf{r}, \mathbf{s})=O(\mathbf{r}, \mathbf{s})
$$

As discussed above, under the transformations $\mathbf{r} \rightarrow-\mathbf{r}$ and $\mathbf{s} \rightarrow-\mathbf{s}$, the decorated dipole operators with even number of transverse indices are invariant and the ones with odd number of indices flip sign. Using these symmetry properties, it is straight forward to show that the strict eikonal and next-to-nextto-eikonal contributions to the numerator of SSA vanish whereas the next-to-eikonal contributions do not vanish. After summing over the gluon polarizations, it can be written as

$$
\begin{aligned}
\left.k^{+}\left(\frac{d \sigma^{\uparrow}}{d k^{+} d^{2} \mathbf{k}}-\frac{d \sigma^{\downarrow}}{d k^{+} d^{2} \mathbf{k}}\right)\right|_{\mathrm{NE}} & =2 \frac{L^{+}}{k^{+}} \int \frac{d^{2} \mathbf{q}}{(2 \pi)^{2}} \varphi_{p}(\mathbf{q}) \mathbf{q}^{2} \int d^{2} \mathbf{r} e^{i \mathbf{r} \cdot(\mathbf{q}-\mathbf{k})} \\
& \times C^{i}(\mathbf{k}, \mathbf{q})\left\{\tilde{C}^{j i}(\mathbf{k}, \mathbf{q}) O_{[0,1]}^{j}(\mathbf{r}, \mathbf{s})-i \frac{\mathbf{q}^{i}}{\mathbf{q}^{2}} O_{[1,0]}(\mathbf{r}, \mathbf{s})\right\} .
\end{aligned}
$$




\section{Conclusions}

We have presented a systematic expansion of the retarded gluon propagator in a background field. The corrections associated with the finite width of the target were calculated at next-to-next-to-eikonal accuracy. These corrections involve new operators referred to as decorated Wilson lines since they include the gradients of the background field along the longitudinal extend of the target. The resulting gluon background propagator is then used to calculate two different observables within the CGC framework: the single inclusive gluon production cross section and the single transverse spin asymmetry for a transversely polarized target. For the single inclusive gluon cross section, it has been shown that the next-to-eikonal corrections vanish and the first non-vanishing contribution to the strict eikonal limit that appear at next-to-next-to-eikonal order have been calculated. On the other hand, for the single transverse spin asymmetry for a transversely polarized target, it has been shown that both strict eikonal terms and next-to-next-to-eikonal terms vanish and the leading contribution to this observable turns out to be next-to-eikonal ones.

\section{References}

[1] F. Gelis, E. Iancu, J. Jalilian-Marian and R. Venugopalan, Ann. Rev. Nucl. Part. Sci. 60, 463 (2010) [arXiv:1002.0333 [hep-ph]].

[2] T. Altinoluk, N. Armesto, G. Beuf, M. Martinez and C. A. Salgado, JHEP 1407, 068 (2014) [arXiv:1404.2219 [hep-ph]].

[3] T. Altinoluk, N. Armesto, G. Beuf and A. Moscoso, arXiv:1505.01400 [hep-ph].

[4] E. Laenen, G. Stavenga and C. D. White, JHEP 0903, 054 (2009) [arXiv:0811.2067 [hep-ph]].

[5] E. Laenen, L. Magnea, G. Stavenga and C. D. White, JHEP 1101, 141 (2011) [arXiv:1010.1860 [hep-ph]].

[6] R. Baier, Y. L. Dokshitzer, A. H. Mueller, S. Peigne and D. Schiff, Nucl. Phys. B 483, 291 (1997) [hep-ph/9607355].

[7] B. G. Zakharov, JETP Lett. 63, 952 (1996) [hep-ph/9607440].

[8] B. G. Zakharov, Phys. Atom. Nucl. 61, 838 (1998) [Yad. Fiz. 61, 924 (1998)] [hep-ph/9807540].

[9] T. Altinoluk and A. Dumitru, arXiv:1512.00279 [hep-ph]. 